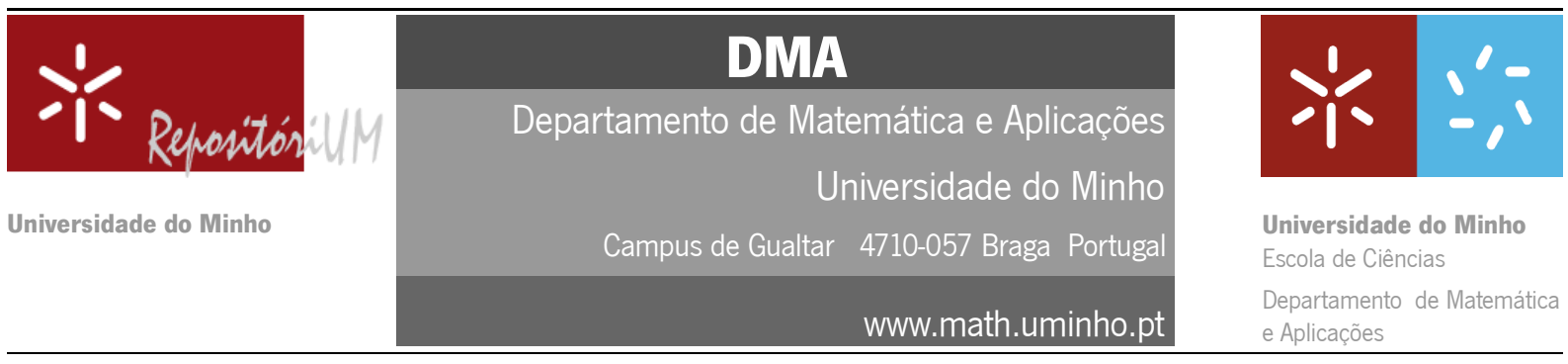

\title{
3D Mappings by Generalized Joukowski Transformations
}

\author{
Carla Cruz ${ }^{a}$ \\ M.I. Falcão ${ }^{b}$ \\ H.R. Malonek ${ }^{a}$
}

${ }^{a}$ Departamento de Matemática, Universidade de Aveiro, Portugal

${ }^{b}$ Departamento de Matemática e Aplicações, Universidade do Minho, Portugal

\section{Information}

\section{Keywords:}

Generalized Joukowski transformation, quasiconformal mappings, hypercomplex differentiable functions.

\author{
Original publication: \\ Computational Science and its Applications, Lecture \\ Notes in Computer Science, vol. 6784, pp. 358-373, \\ 2011 \\ DOI: $10.1007 / 978-3-642-21931-3 \_28$ \\ www.springerlink.com
}

\begin{abstract}
The classical Joukowski transformation plays an important role in different applications of conformal mappings, in particular in the study of flows around the so-called Joukowski airfoils. In the 1980s H. Haruki and M. Barran studied generalized Joukowski transformations of higher order in the complex plane from the view point of functional equations. The aim of our contribution is to study the analogue of those generalized Joukowski transformations in Euclidean spaces of arbitrary higher dimension by methods of hypercomplex analysis. They reveal new insights in the use of generalized holomorphic functions as tools for quasi-conformal mappings. The computational experiences focus on 3D-mappings of order 2 and their properties and visualizations for different geometric configurations, but our approach is not restricted neither with respect to the dimension nor to the order.
\end{abstract}

\section{Introduction and Notations}

First of all we refer some basic notations used in hypercomplex analysis. Let $\left\{e_{1}, e_{2}, \ldots, e_{m}\right\}$ be an orthonormal basis of the Euclidean vector space $\mathbb{R}^{m}$ with the non-commutative product according to the multiplication rules $e_{k} e_{l}+e_{l} e_{k}=-2 \delta_{k l}, k, l=1, \ldots, m$, where $\delta_{k l}$ is the Kronecker symbol. The set $\left\{e_{A}: A \subseteq\{1, \ldots, m\}\right\}$ with $e_{A}=e_{h_{1}} e_{h_{2}} \ldots e_{h_{r}}, 1 \leq h_{1}<\cdots<h_{r} \leq m, e_{\emptyset}=e_{0}=1$, forms a basis of the $2^{m}$-dimensional Clifford algebra $\mathcal{C} \ell_{0, m}$ over $\mathbb{R}$. Let $\mathbb{R}^{m+1}$ be embedded in $\mathcal{C} \ell_{0, m}$ by identifying $\left(x_{0}, x_{1}, \ldots, x_{m}\right) \in \mathbb{R}^{m+1}$ with the algebra's element $x=x_{0}+\underline{x} \in \mathcal{A}:=\operatorname{span}_{\mathbb{R}}\left\{1, e_{1}, \ldots, e_{m}\right\} \subset \mathcal{C} \ell_{0, m}$. The elements of $\mathcal{A}$ are called paravectors and $x_{0}=\operatorname{Sc}(x)$ and $\underline{x}=\operatorname{Vec}(x)=e_{1} x_{1}+\cdots+e_{m} x_{m}$ are the scalar resp. vector part of the paravector $x$. The 
conjugate of $x$ is given by $\bar{x}=x_{0}-\underline{x}$ and the norm $|x|$ of $x$ is defined by $|x|^{2}=x \bar{x}=\bar{x} x=x_{0}^{2}+x_{1}^{2}+\cdots+x_{m}^{2}$. Consequently, any non-zero $x$ has an inverse defined by $x^{-1}=\frac{\bar{x}}{|x|^{2}}$.

We consider functions of the form $f(z)=\sum_{A} f_{A}(z) e_{A}$, where $f_{A}(z)$ are real valued, i.e. $\mathcal{C} \ell_{0, m^{-}}$-valued functions defined in some open subset $\Omega \subset \mathbb{R}^{m+1}$. Continuity and real differentiability of $f$ in $\Omega$ are defined componentwise. The generalized Cauchy-Riemann operator in $\mathbb{R}^{m+1}, m \geq 1$, is defined by

$$
\bar{\partial}:=\partial_{0}+\partial_{\underline{x}}
$$

where

$$
\partial_{0}:=\frac{\partial}{\partial x_{0}}, \quad \partial_{\underline{x}}:=e_{1} \frac{\partial}{\partial x_{1}}+\cdots+e_{m} \frac{\partial}{\partial x_{m}} .
$$

The higher dimensional analogue of an holomorphic function is usually defined as $\mathscr{C}^{1}(\Omega)$-function $f$ satisfying the equation $\bar{\partial} f=0$ (resp. $f \bar{\partial}=0$ ) which is the hypercomplex form of a generalized Cauchy-Riemann system. By historical reasons it is called left monogenic (resp. right monogenic) [3]. An equivalent definition of monogenic functions is that $f$ is hypercomplex differentiable in $\Omega$ in the sense of [9], [16], i.e. that for $f$ exists a uniquely defined areolar derivative $f^{\prime}$ in each point of $\Omega$ (see also [18]). Then $f$ is automatically real differentiable and $f^{\prime}$ can be expressed by the real partial derivatives as $f^{\prime}=1 / 2 \partial f$, where $\partial:=\left(\partial_{0}-\partial_{x}\right)$ is the conjugate Cauchy-Riemann operator. Since a hypercomplex differentiable function belongs to the kernel of $\bar{\partial}$, it follows that in fact $f^{\prime}=\partial_{0} f=-\partial_{\underline{x}} f$ like in the complex case. Due to the role of the complex derivative in the study of conformal transformations in $\mathbb{C}$, it was natural to investigate the role of the hypercomplex derivative from the view point of quasi-conformal mappings in $\mathbb{R}^{m+1}[17,20]$. Indeed, conformal mappings in real Euclidean spaces of dimension higher than 2 are restricted to Möbius transformations (Liouville's theorem) which are not monogenic functions. But obviously, this does not mean that monogenic functions cannot play an important role in applications to the more general class of quasi-conformal mappings, intensively studied by real and several complex variable methods so far. The advantage of hypercomplex methods applicable to Euclidean spaces of arbitrary real dimensions (not only of even dimensions like in the case of $\mathbb{C}^{n}$-methods) is already evident for one of the most important case in practical applications, i.e. the lowest odd dimensional case of $\mathbb{R}^{2+1}$. Besides other practical reasons, it still allows directly visualization of all geometric mapping properties. Of course, quasi-conformal 3D-mappings demand more computational capacities than conformal 2D-mappings. But since hypercomplex analysis methods are developed in analogy with complex methods $([2,4,6,10,11,12,19])$, the expectations on their efficiency for solving 3D-mapping problems are in general very high. Nevertheless, a systematical work on this subject is still missing, presumably because of missing familiarity with hypercomplex methods and their use in practical problems. Therefore we intend to give some insights in this subject also by comparison with the complex 2D case and by 2D- and 3D-plots produced with Mathematica for their visualization. Worth noticing that in this paper only basic computational aspects are discussed. A deeper function theoretic analysis, for instance, the relationship of the hypercomplex derivative and the Jacobian matrix, was not the aim of this work. However, in [5], the reader can find the corresponding results for the hypercomplex case of generalized Joukowski transformations of order $k=1$.

\section{Generalized Joukowski Transformations in the Complex Plane}

In [13] and later in [1] H. Haruki and M. Barran studied specific functional equations whose unique solution is given by

$$
\widetilde{w}=\widetilde{w}(z)=\frac{1}{2}\left(z^{k}+z^{-k}\right),
$$

where $k$ is a positive integer. The function $\widetilde{w}=w_{0}+i w_{1}$ is said to be a generalized Joukowski transformation of order $k$. It maps the unit circle into the interval $[-1,1]$ of the real axis in the $\widetilde{w}$-plane traced $2 k$ times. Obviously there is no essential difference between transforming the unit circle into the real interval $[-1,1]$ and transforming it into the imaginary interval $[-i, i]$. If we use modified polar coordinates ${ }^{1}$ in the form

\footnotetext{
${ }^{1}$ For $k=1$ see [5] or [6], where this modified treatment of the Joukowski transformation was used for the first time. It allows to connect the 2D case more directly with the corresponding hypercomplex $3 \mathrm{D}$ case, where the unit sphere $S^{2}$ has a purely vector-valued image in analogy to the purely imaginary image of $S^{1}$.
} 
$z=\rho e^{i\left(\frac{\pi}{2}-\varphi\right)}=\rho(\sin \varphi+i \cos \varphi)$, for $\varphi \in[0,2 \pi]$, then we obtain the interval $[-i, i]$ as the image of the unit circle $S^{1}$ under the mapping

$$
w=w(z)=\frac{1}{2}\left(z^{k}-z^{-k}\right) .
$$

Moreover the real and imaginary parts of $w$ are obtained in the following form

$$
w_{0}=\frac{1}{2}\left(\rho^{k}-\frac{1}{\rho^{k}}\right) \cos \left(\frac{k}{2} \pi-k \varphi\right), \quad w_{1}=\frac{1}{2}\left(\rho^{k}+\frac{1}{\rho^{k}}\right) \sin \left(\frac{k}{2} \pi-k \varphi\right) .
$$

Circles of radius $\rho \neq 1$ are transformed onto confocal ellipses with semi-axis

$$
a=\frac{1}{2}\left|\rho^{k}-\frac{1}{\rho^{k}}\right|, \quad b=\frac{1}{2}\left(\rho^{k}+\frac{1}{\rho^{k}}\right)
$$

and foci $w=i$ and $w=-i$.

Figures 1 and 2 show the well known images of disks with radii equal or greater than one under the mapping $w$, for $k=1$. To stress the double covering of the segment $[-i, i]$, in the case $\rho=1$, we present the images of both semi-disks separately. The restriction to black and white figures suggested to use also dotted lines. The same is analogously done for $k=2$ with dashed and dotted lines. Figures 3 and 4 are the $k=2$ analogue of Fig. 1 and 2. We underline the fact that, in this case, the mapping function is 4 -fold when $\rho=1$ and 2 -fold for $\rho>1$.

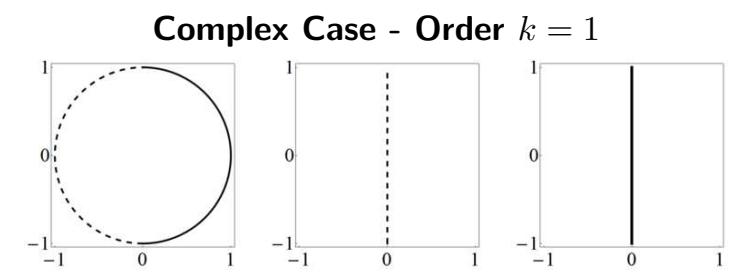

Figure 1: The two unit semi-disks and their corresponding images
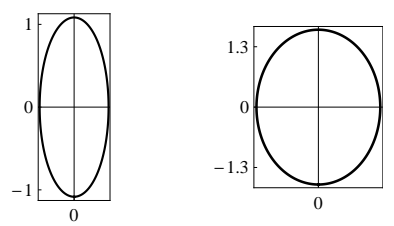

Figure 2: The images of disks of radius $\rho=1.5$ and $\rho=3$

\section{Generalized Joukowski Transformations in $\mathbb{R}^{m+1}$}

In [5] the higher dimensional analogue of the classical Joukowski transform for $m \geq 1$ and $k=1$ has been studied in detail for the first time. For its generalization to the case of arbitrary order $k \geq 1$, we apply two monogenic paravector-valued functions which generalize $z^{k}$ and $z^{-k}$ in $\mathbb{C}$. They are defined for $m \geq 1$ by

$$
\mathcal{P}_{k}^{m}(x)=\sum_{s=0}^{k} c_{s}(m)\left(\begin{array}{l}
k \\
s
\end{array}\right) x_{0}^{k-s} \underline{x}^{s}=\sum_{s=0}^{k} T_{s}^{k}(m) x^{k-s} \bar{x}^{s}
$$

and

$$
E_{m}(x)=\frac{\bar{x}}{|x|^{m+1}}
$$

where $c_{k}(m)=\sum_{s=0}^{k}(-1)^{s} T_{s}^{k}(m)$ and

$$
T_{s}^{k}(m)=\frac{k !}{m_{(k)}} \frac{\left(\frac{m+1}{2}\right)_{(k-s)}\left(\frac{m-1}{2}\right)_{(s)}}{(k-s) ! s !},
$$


Complex Case - Order $k=2$
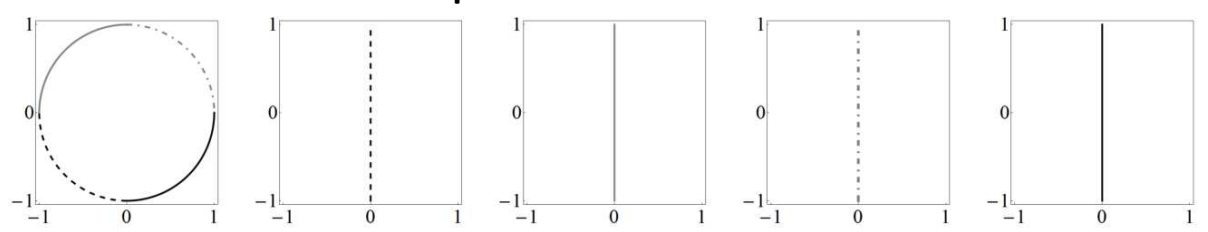

Figure 3: The images of the quarter-disks
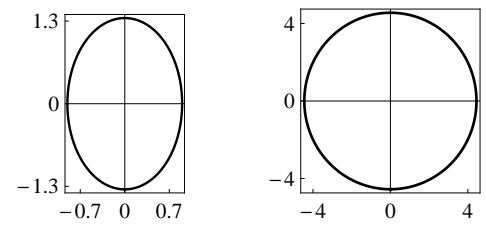

Figure 4: The images of semi-disks of radius $\rho=1.5$ and $\rho=3$

with $m_{(k)}$ denoting the Pochhammer symbol. Properties of the polynomials $\mathcal{P}_{k}^{m}(x)$, which form an example of a hypercomplex Appell sequence, as well as of the fundamental solution $E_{m}(x)$ of the generalized CauchyRiemann system (see Sect. 1) can be found in [7], respectively [8].

Analogously to [5], the proposed higher dimensional analogue of the Joukowski transformation is given by

Definition 1. Let $x=x_{0}+\underline{x} \in \mathcal{A} \cong \mathbb{R}^{m+1} \subset \mathcal{C} \ell_{0, m}$. The generalized hypercomplex Joukowski transformation of order $k$ is defined as

$$
J_{k}^{m}(x)=\alpha_{k}\left(\mathcal{P}_{k}^{m}(x)+\frac{(-1)^{k}}{m_{(k-1)}} E_{m}^{(k-1)}(x)\right),
$$

where $\alpha_{k}$ is a real constant and $E_{m}^{(k-1)}(x)$ denotes the hypercomplex derivative of order $(k-1)$, for $k \geq 1$.

Formula (5) with $\alpha_{1}=\frac{2}{3}$ is the generalized hypercomplex Joukowski transformation considered in [5]. In fact, (5) can be expressed only in terms of those monogenic polynomials of type $\mathcal{P}_{k}^{m}$, if we use the Kelvin transform for harmonic functions in several real variables. The connection of monogenic functions with harmonic functions relies on the fact that monogenic functions are also harmonic functions, since the Laplace operator for $(m+1)$-real variables is factorized by the generalized Cauchy-Riemann operator $\bar{\partial}$ and its conjugate operator $\partial$ (see Sect. 1 ) in the form $\Delta=\bar{\partial} \partial$. Often this property, well known from Complex Analysis, is considered as the essential reason why hypercomplex analysis or, more general Clifford Analysis (see the title of [3]), could be considered as a refinement of Harmonic Analysis. For our purpose we adapt the notation of [8] and use

Definition 2. Given a monogenic, paravector-valued, and homogeneous function $f$ of degree $k$, then the monogenic homogeneous function of degree $-(k+m)$ defined in $\mathbb{R}^{m+1} \backslash\{0\}$

$$
I[f](x):=E_{m}(x) f\left(x^{-1}\right)
$$

is called the Kelvin transform of $f$.

The Kelvin transform of an harmonic function generalizes the inversion on the unit circle in the complex plane to the inversion on the unit sphere in $\mathbb{R}^{m+1}$ (more about its properties and applications in hypercomplex analysis can be found in [8]). The following proposition shows the connection between the Kelvin transform applied to the polynomials $\mathcal{P}_{k}^{m}$ and the hypercomplex derivative of $E_{m}$.

Proposition 1. Let $\mathcal{P}_{k}^{m}$ and $E_{m}$ be the functions defined by (3) and (4) respectively. Then

$$
E_{m}^{(k)}(x)=(-1)^{k} m_{(k)} I\left[\mathcal{P}_{k}^{m}\right](x) .
$$


Proof. The factorization of the fundamental solution in the form

$$
\frac{\bar{x}}{|x|^{m+1}}=\left(\frac{\bar{x}}{|x|^{2}}\right)^{\frac{m+1}{2}}\left(\frac{x}{|x|^{2}}\right)^{\frac{m-1}{2}}
$$

allows the use of Leibniz' differentiation rule in order to obtain

$$
\begin{aligned}
E_{m}^{(k)}(x) & =\frac{\partial^{k}}{\partial x_{0}^{k}} \frac{\bar{x}}{|x|^{m+1}} \\
& =\sum_{s=0}^{k}\left(\begin{array}{l}
k \\
s
\end{array}\right)(-1)^{k}\left(\frac{m+1}{2}\right)_{(k-s)}\left(\frac{m-1}{2}\right)_{(s)}\left(\frac{\bar{x}}{|x|^{2}}\right)^{\frac{m+1}{2}+k-s}\left(\frac{x}{|x|^{2}}\right)^{\frac{m-1}{2}+s} \\
& =(-1)^{k} k ! \frac{\bar{x}}{|x|^{m+2 k+1}} \sum_{s=0}^{k} \frac{\left(\frac{m+1}{2}\right)_{(k-s)}}{(k-s) !} \frac{\left(\frac{m-1}{2}\right)_{(s)}}{s !} \bar{x}^{k-s} x^{s} \\
& =(-1)^{k} m_{(k)} \frac{\bar{x}}{|x|^{m+2 k+1}} \sum_{s=0}^{k} T_{s}^{k}(m) \bar{x}^{k-s} x^{s} \\
& =(-1)^{k} m_{(k)} \frac{\bar{x}}{|x|^{m+2 k+1}} \mathcal{P}_{k}^{m}(\bar{x})
\end{aligned}
$$

On the other hand, recalling that the polynomials $\mathcal{P}_{k}^{m}$ have the property of being homogeneous of degree $k$ and applying the Kelvin transform (6) we obtain

$$
I\left[\mathcal{P}_{k}^{m}\right](x)=\frac{\bar{x}}{|x|^{m+1}} \mathcal{P}_{k}^{m}\left(\frac{\bar{x}}{|x|^{2}}\right)=\frac{\bar{x}}{|x|^{m+2 k+1}} \mathcal{P}_{k}^{m}(\bar{x})
$$

and the final result follows now at once.

For $m=1$, i.e. in the complex case, we have $I\left[z^{k}\right]=z^{-(k+1)}$ and $E_{1}^{(k)}(z)=\left(z^{-1}\right)^{(k)}$ and the factor in (7) is nothing else than $(-1)^{k} k$ !. Notice that this corresponds to the form (2) of the classical Joukowski transformation which we are using. Moreover, formula (5) shows a generalization of the hypercomplex Joukowski transformation studied in [5] and [6] by means of the fundamental solution $E_{m}$. Proposition 1 and, in particular, formula (9) simplifies the necessary calculations since we can rely on the well studied structure of the polynomials $\mathcal{P}_{k}^{m}$.

In what follows we focus on the case $m=2$, i.e. $\mathbb{R}^{3}$, and write briefly $\mathcal{P}_{k}^{2}(x)=\mathcal{P}_{k}(x), J_{k}^{2}(x)=J_{k}(x)$ and $c_{s}(2)=c_{s}$. This means, that we consider now for arbitrary $k \geq 1$ the generalized hypercomplex Joukowski transformation in the form

$$
J_{k}(x)=\alpha_{k}\left(\mathcal{P}_{k}(x)+\frac{(-1)^{k}}{k !} E^{(k-1)}(x)\right)=\alpha_{k}\left(\mathcal{P}_{k}(x)-I\left[\mathcal{P}_{k-1}\right](x)\right) .
$$

Then the image of the unit sphere $S^{2}=\left\{x=x_{0}+\underline{x}:|x|^{2}=1\right\}$ under the mapping $J_{k}$ is given by:

$$
\begin{aligned}
J_{k}\left(S^{2}\right) & =\alpha_{k} \sum_{s=0}^{k} c_{s}\left(\begin{array}{c}
k \\
s
\end{array}\right) x_{0}^{k-s} \underline{x}^{s}-\alpha_{k}\left(x_{0}-\underline{x}\right) \sum_{s=0}^{k-1}(-1)^{s} c_{s}\left(\begin{array}{c}
k-1 \\
s
\end{array}\right) x_{0}^{k-1-s} \underline{x}^{s} \\
& =\alpha_{k}\left(c_{k}+(-1)^{k-1} c_{k-1}\right) \underline{x}^{k}+A(k, s),
\end{aligned}
$$

where

$$
A(k, s)=\alpha_{k} \sum_{s=1}^{k-1}\left\{c_{s}\left[\left(\begin{array}{l}
k \\
s
\end{array}\right)+(-1)^{s-1}\left(\begin{array}{c}
k-1 \\
s
\end{array}\right)\right]+(-1)^{s-1} c_{s-1}\left(\begin{array}{c}
k-1 \\
s-1
\end{array}\right)\right\} x_{0}^{k-s} \underline{x}^{s} .
$$

Here, as well as before, the constant $\alpha_{k}$ remains for the moment undefined. Applying now the fact that the coefficients $c_{k}$ in this special case of $m=2$ (cf. [7]) are equal to

$$
c_{k}= \begin{cases}\frac{k ! !}{(k+1) ! !}, & \text { if } k \text { is odd } \\ c_{k-1}, & \text { if } k \text { is even }\end{cases}
$$


we see that the coefficients of $x_{0}^{k-s} \underline{x}^{s}$, for $0 \leq s \leq k$ in (10) depend on the parity of $s$. Using (11) together with well known properties of the binomial coefficients one obtains easily the following identity

$$
c_{s}\left[\left(\begin{array}{c}
k \\
s
\end{array}\right)+(-1)^{s-1}\left(\begin{array}{c}
k-1 \\
s
\end{array}\right)\right]+(-1)^{s-1} c_{s-1}\left(\begin{array}{l}
k-1 \\
s-1
\end{array}\right)= \begin{cases}c_{s}\left(\begin{array}{l}
k \\
s
\end{array}\right) \frac{2 k+1}{k}, & \text { if } s \text { is odd } \\
0, & \text { if } s \text { is even. }\end{cases}
$$

Finally the image of the unit sphere for $k>1$ is given by:

$$
J_{k}\left(S^{2}\right)=\alpha_{k} \frac{2 k+1}{k} \underline{x} \sum_{l=0}^{\left[\frac{k-1}{2}\right]} c_{2 l+1}\left(\begin{array}{c}
k \\
2 l+1
\end{array}\right) x_{0}^{k-(2 l+1)} \underline{x}^{2 l}
$$

Since $\underline{x}^{2 l}=(-1)^{l}|\underline{x}|^{2 l}$ the sum in (12) is real and therefore the unit sphere is mapped onto the hyperplane $w_{0}=0$, or equivalently, $J_{k}\left(S^{2}\right)$ is a paravector with vanishing scalar part, i.e. a pure vector. The same is true for arbitrary $m \geq 3$, but the corresponding proof relies on more difficult expressions of the $c_{k}(m)($ see [7]) and for this reason has been omitted here.

For $k=1,2,3$ we have the following explicit expressions for the image of the unit sphere $S^{2}$ under the mapping $J_{k}$ :

$$
J_{1}\left(S^{2}\right)=\alpha_{1} \frac{3}{2} \underline{x}, \quad J_{2}\left(S^{2}\right)=\alpha_{2} \frac{5}{2} \underline{x} x_{0}, \quad J_{3}\left(S^{2}\right)=\alpha_{3} \frac{7}{3} \underline{x}\left(\frac{15}{8} x_{0}^{2}-\frac{3}{8}\right) .
$$

Until now we did not discuss the role of the constant $\alpha_{k}$ in the general expression of (5). But we have already mentioned the form of the generalized hypercomplex Joukowski transformation for $k=1$ considered in [6], where its value is $\alpha_{1}=\frac{2}{3}$. The reason for such a choice was the standardization of the mapping in such a way, that the image of $S^{2}$ would be the unit circle $S^{1}$ in the hyperplane $w_{0}=0$. Obviously, this corresponds to the unit interval $[-i, i]$ as the image of $S^{1}$ in the complex case. Writing now briefly (12) in the form

$$
J_{k}\left(S^{2}\right)=\alpha_{k} \underline{x} B_{k}(x)
$$

with

$$
B_{k}(x)=B_{k}\left(x_{0}, \underline{x}\right):=\frac{2 k+1}{k} \sum_{l=0}^{\left[\frac{k-1}{2}\right]} c_{2 l+1}\left(\begin{array}{c}
k \\
2 l+1
\end{array}\right) x_{0}^{k-(2 l+1)} \underline{x}^{2 l},
$$

we see that the problem of determining $\alpha_{k}$ for each value of $k$ in the previously mentioned way is solved by

$$
\alpha_{k}:=\left(\max _{|x|^{2}=1} B_{k}\left(x_{0}, \underline{x}\right)\right)^{-1} .
$$

From Sect. 2 we recall the use of modified polar coordinates in the complex plane case for describing the classical Joukowski transformation with the purely imaginary interval $[-i, i]$ as the image of the unit circle. They lead to the application of geographic spherical coordinates in the 3-dimensional space and allow to describe easily the mapping properties of $J_{1}$ as explained in [5] and [6]. Therefore, let $(\rho, \varphi, \theta)$ be radius, latitude, and longitude respectively, so that we work with

$$
x_{1}=\rho \cos \varphi \cos \theta, \quad x_{2}=\rho \cos \varphi \sin \theta, \quad x_{0}=\rho \sin \varphi
$$

where $\rho>0,-\pi<\theta \leq \pi$ and $-\frac{\pi}{2} \leq \varphi \leq \frac{\pi}{2}$. Thus, from (13) we have in terms of spherical coordinates

$$
\left|J_{1}\left(S^{2}\right)\right|^{2}=\alpha_{1}^{2}\left(\frac{3}{2}\right)^{2} \cos ^{2} \varphi
$$

This shows that the unit disk $S^{1}$ in the hyperplane $w_{0}=0$ as the image of the unit sphere $S^{2}$ in $\mathbb{R}^{3}$ is obtained if $\alpha_{1}$ is chosen equal to $\frac{2}{3}([5])$. Analogously, for $k=2$ we have that

$$
\begin{aligned}
\left|J_{2}\left(S^{2}\right)\right|^{2} & =\alpha_{2}^{2}\left(\frac{5}{2}\right)^{2} \cos ^{2} \varphi \sin ^{2} \varphi \\
& =\alpha_{2}^{2}\left(\frac{5}{4}\right)^{2} \sin ^{2}(2 \varphi) .
\end{aligned}
$$


Again, it is easy to see that in this case $\alpha_{2}=\frac{4}{5}$ guarantees the desired mapping. In the same way it is, in principle, possible to determine for every $k$ the corresponding value of $\alpha_{k}$ in form of (14). Since the solution of algebraic equations of higher order becomes involved, it will obviously be more complicated than in those lower dimensional cases.

\section{3D Mappings by Generalized Joukowski Transformations}

After the discussion of the basic analytical aspects of generalized hypercomplex Joukowski transformations, we now focus on some basic geometric mapping aspects of the transformations $J_{1}$ and $J_{2}$ in $\mathbb{R}^{3}$. Our special concern are properties similar (or not) to those of the complex case. Using, as referred in the previous section, the normalization factor $\alpha_{1}=2 / 3$ in (5) we obtain the components of $J_{1}=w_{0}+w_{1} e_{1}+w_{2} e_{2}$ in terms of spherical coordinates in the form of

$$
\begin{aligned}
& w_{0}=\frac{2}{3}\left(1-\frac{1}{\rho^{3}}\right) \rho \sin \varphi \\
& w_{1}=\frac{2}{3}\left(\frac{1}{2}+\frac{1}{\rho^{3}}\right) \rho \cos \varphi \cos \theta \\
& w_{2}=\frac{2}{3}\left(\frac{1}{2}+\frac{1}{\rho^{3}}\right) \rho \cos \varphi \sin \theta .
\end{aligned}
$$

One can easily observe that $J_{1}$ maps spheres of radius $\rho \neq 1$ into spheroids with equatorial radius $a=$ $\frac{2}{3} \rho\left(\frac{1}{2}+\frac{1}{\rho^{3}}\right)$ and polar radius $b=\frac{2}{3} \rho\left|1-\frac{1}{\rho^{3}}\right|$. But if $\rho=1$ then (15)-(17) implies that $J_{1}\left(S^{2}\right)$ has a real part identically zero and satisfies

$$
w_{1}^{2}+w_{1}^{2}=\cos ^{2} \varphi
$$

which means that the two-fold unit disk in the hyperplane $w_{0}=0$ is the image of the unit sphere. Figure 5 shows the images of both hemispheres with radius equal to one under the mapping $J_{1}$. The relations between the polar and the equatorial radius show also that the corresponding image, for values of $\rho<\sqrt[3]{4}$, is an oblate spheroid and for values of $\rho>\sqrt[3]{4}$ is a prolate spheroid, whereas the value of $\rho=\sqrt[3]{4}$ corresponds to a sphere. Moreover, as examples for comparison with the complex case in Sect. 2, Fig. 6 shows the images of spheres with different radii greater than one, in particular the sphere obtained as image of a sphere with $\rho=\sqrt[3]{4}$. This limit case between images of an oblate and a prolate spheroid was for the first time determined in [5]. These pictures reveal the similarity between the complex and the hypercomplex cases, but different from the complex case where the type of ellipses remains the same for all $\rho>1$ (see Fig. 2).

Consider now the case $k=2$ for which we use the generalized hypercomplex Joukowski transformation with $\alpha_{2}=\frac{4}{5}$ as explained in the previous section, i.e.

$$
J_{2}(x)=\frac{4}{5}\left(\mathcal{P}_{2}(x)+\frac{1}{2} E^{\prime}(x)\right)=\frac{4}{5}\left(x_{0}^{2}+x_{0} \underline{x}+\frac{1}{2} \underline{x}^{2}\right)+\frac{2}{5}\left(\frac{x_{0}-\underline{x}}{|x|^{3}}\right)^{\prime}
$$

Its real components have, in terms of spherical coordinates, the following expressions:

$$
\begin{aligned}
w_{0} & =\frac{2}{5}\left(1-\frac{1}{\rho^{5}}\right) \rho^{2}\left(-1+3 \sin ^{2} \varphi\right) \\
w_{1} & =\frac{4}{5}\left(1+\frac{3}{2 \rho^{5}}\right) \rho^{2} \sin \varphi \cos \varphi \cos \theta \\
w_{2} & =\frac{4}{5}\left(1+\frac{3}{2 \rho^{5}}\right) \rho^{2} \sin \varphi \cos \varphi \sin \theta
\end{aligned}
$$

As we expected, spheres in $\mathbb{R}^{3}$ with radius $\rho \neq 1$ are transformed into spheroids, but this time, we obtain a 2 -fold mapping. It is also possible to detect another new property, different from the previous case $k=1$, namely the effect that the center of the spheroids does not anymore remain on the origin. The shift of the 


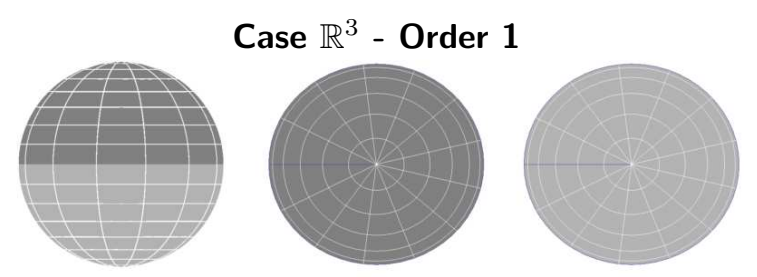

Figure 5: The images of both hemispheres of $S^{2}$
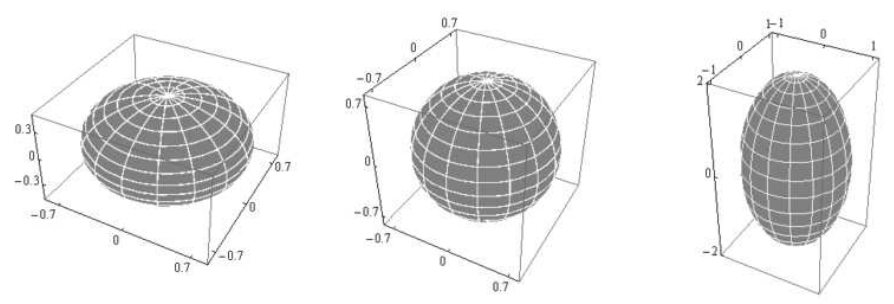

Figure 6: The images of spheres of radius $\rho=1.3, \rho=\sqrt[3]{4}$ and $\rho=3$

center from the origin occurs in direction of the real $w_{0}$-axis and is equal to $\frac{1}{5} \rho^{2}\left(1-\frac{1}{\rho^{5}}\right)$. Therefore the polar radius is given by $b=\frac{3}{5} \rho^{2}\left|1-\frac{1}{\rho^{5}}\right|$ and the equatorial radius by $a=\frac{1}{5} \rho^{2}\left(2+\frac{3}{\rho^{5}}\right)$, so that we have:

$$
\frac{\left[w_{0}-\frac{1}{5} \rho^{2}\left(1-\frac{1}{\rho^{5}}\right)\right]^{2}}{\left[\frac{3}{5} \rho^{2}\left(1-\frac{1}{\rho^{5}}\right)\right]^{2}}+\frac{w_{1}^{2}}{\left[\frac{1}{5} \rho^{2}\left(2+\frac{3}{\rho^{5}}\right)\right]^{2}}+\frac{w_{2}^{2}}{\left[\frac{1}{5} \rho^{2}\left(2+\frac{3}{\rho^{5}}\right)\right]^{2}}=1 .
$$

The following proposition summarizes some properties of the mapping $J_{2}$ :

\section{Proposition 2.}

1. Spheres with radius $1<\rho<\sqrt[5]{6}$ are 2 -folded transformed into oblate spheroids.

2. The sphere with radius $\rho=\sqrt[5]{6}$ is 2 -folded transformed into the sphere with center $\left(0,0, \frac{1}{\sqrt[5]{6^{3}}}\right)$.

3. Spheres with radius $\sqrt[5]{6}<\rho$ are 2-folded transformed into prolate spheroids.

4. The unit sphere $S^{2}$ is 4-folded mapped onto the unit circle (including its interior) in the hyperplane $w_{0}=0$.

Figure 7 shows the images of four zones of the unit sphere under the mapping $J_{2}$ as consequence of the 4-fold mapping of the unit sphere $S^{2}$ to $S^{1}$ (cf. with the 2 -fold mapping in the case $k=1$ in Fig. 5). Analogously to $k=1$ where we have shown images of spheres in Fig. 6, the images in Fig. 8 are the result of mapping one of the hemispheres with several radius greater than one. Similar to the case $k=1$ the value $\rho=\sqrt[5]{6}$ gives a sphere but now not centered at the origin.

\section{Final Remarks on Joukowski Type 3D Airfoils}

The classical Joukowski transformation (1), or equivalently (2), plays in Aerodynamics an important role in the study of flows around so-called Joukowski airfoils, since it maps circles with centers sufficiently near to the origin into airfoils. In the classical Dictionary of Conformal Representations [15], for example, or the more recent book Computational Conformal Mapping [14], specially dedicated to computational aspects, one can 


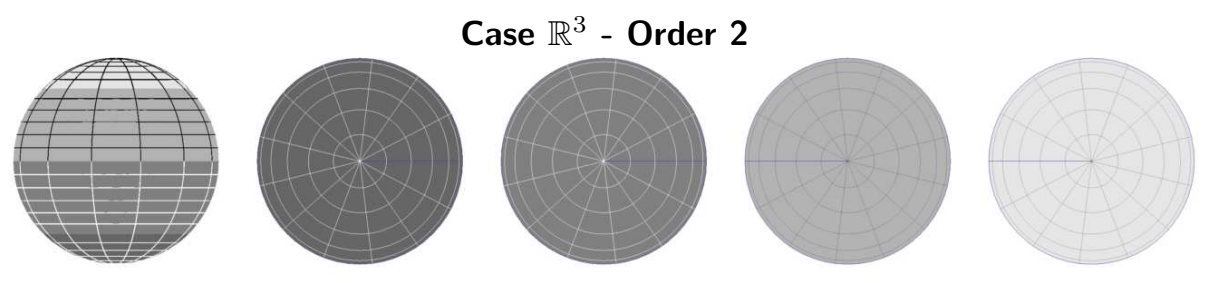

Figure 7: The images of the unit sphere
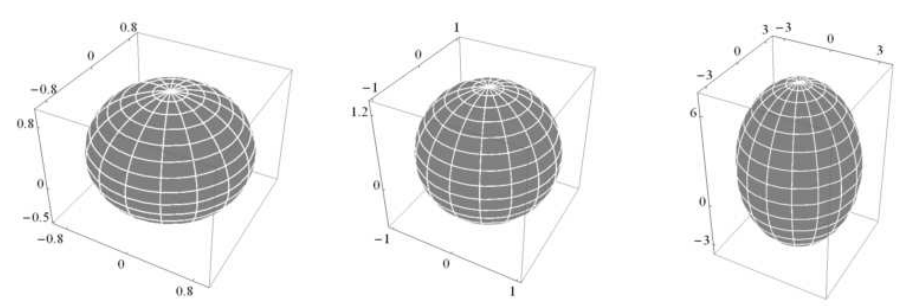

Figure 8: The images of hemispheres of radius $\rho=1.3, \rho=\sqrt[5]{6}$ and $\rho=3$

find a lot of details about those symmetric or unsymmetric airfoils. They are, for $k=1$, images in the $w$-plane obtained by mapping functions of the form (1) of circles centered at a point sufficiently near to the origin and passing through -1 or $1 .^{2}$ For example, Fig. 9 shows two symmetric airfoils that are images of circles centered at points (different from the origin) on the imaginary axis in the complex plane. An unsymmetric (and more interesting for studies in aerodynamics) Joukowski airfoil as image of a circle centered at a point in the first quadrant is shown in Fig. 10.

In the hypercomplex case, the paper [6] analogously includes images produced with Maple of spheres in $\mathbb{R}^{3}$ centered at points of one of the axes $x_{1}$ or $x_{2}$ with a small displacement and passing through the endpoints of the unit vectors $e_{1}$ and $e_{2}$, respectively. We reproduce them in Fig. 11. Here we show in Fig. 12 the image of a sphere with $\rho>1$ in a more general position. More concretely, its center is chosen in $(0.1,0.1,0)$ and the point $(-1 / 2 \sqrt{2},-1 / 2 \sqrt{2}, 0)$ is the corresponding fixpoint of the mapping. Though both cases are images of dislocated from the origin spheres of the same radius $\rho>1$, it seems that the direction of the displacement only along one axis or not, for example - leads to slightly different images, as the figures suggest. Particularly one can recognize different curvatures of the surfaces.

It seems to us not presumptuous to interpret those figures as some kind of symmetric Joukowski airfoils generalized to 3D and extended in different directions. Figure 13 which shows some cuts of the domain illustrated in Fig. 12 parallel to the hyperplane $w_{1}=w_{2}$ is in our opinion a very clear illustration of this situation. If the displacement of the center of the sphere is also done in all three directions unsymmetrically with three different values of the center coordinates, then we get a mapping like the one presented in the Fig. 14. It could be interpreted as some kind of unsymmetric Joukowski airfoil in 3D.

Finally we compare some mappings for the case $k=2$ in 2D and 3D. Due to the higher order of singularities in the origin we should also be aware of more complicated images of circles and spheres, respectively, with radii different from $\rho=1$ (Figures 15-16). Nevertheless, we would not exclude the possibility, that they could be useful for mathematical models working with more complicated geometric configurations with some singularities, particularly in $\mathbb{R}^{3}$.

Resuming this steps towards a more systematic study of 3D mappings realized by generalized hypercomplex Joukowski transformations we would like to mention that hypercomplex methods seem to us in general a promising tool for quasi-conformal mappings in $\mathbb{R}^{3}([20])$. We could produce by monogenic functions some mappings from the unit sphere in $\mathbb{R}^{3}$ that remind significant similarities in our opinion with one-wing objects reported in connection with an an alternative airframe design, called Blended Wing Body, or BWB (see [21]), which include interesting images of ongoing constructions of one-wing airplanes). There one find the remark that: The advantages of the BWB approach are efficient high-lift wings and a wide airfoil-shaped body. With

\footnotetext{
${ }^{2}$ Applied to our modified form (2) in Sect. 2 they should pass through $-i$ and $i$. Of course, these fixpoints are only chosen for some normalization of the mapping and are not essential for its global behavior.
} 


\section{Complex Case - Order $k=1$}
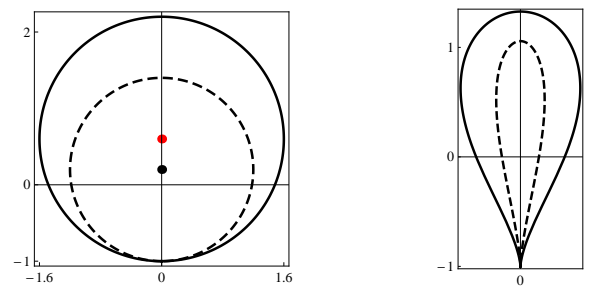

Figure 9: The image of disks with radius $\rho=1.2$ and $\rho=1.6$ centered at $d=(\rho-1) i$
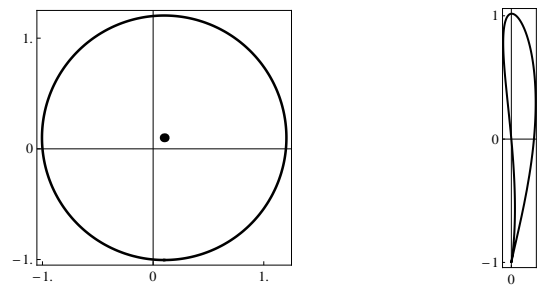

Figure 10: The image of the disk with radius $\rho=|1+d|$ and centered at $d=0.1+0.1 i$

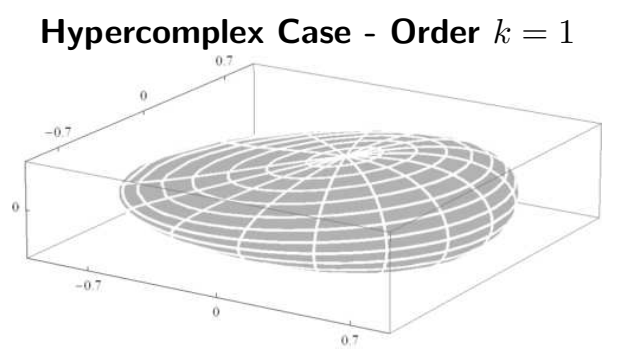

Figure 11: The image of a sphere of radius $\rho=1+|d|$ and center $d=0.1 e_{1}$

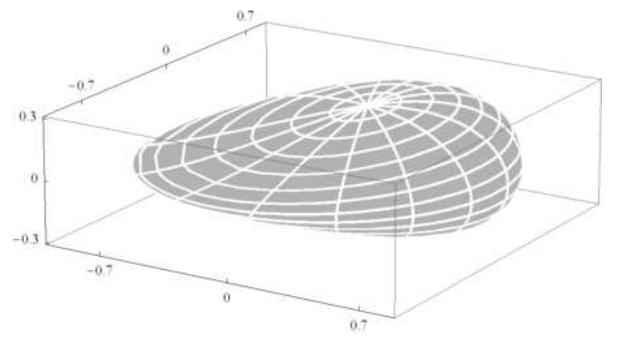

Figure 12: The image of a sphere of radius $\rho=1+|d|$ and center $d=0.1 e_{1}+0.1 e_{2}$ 


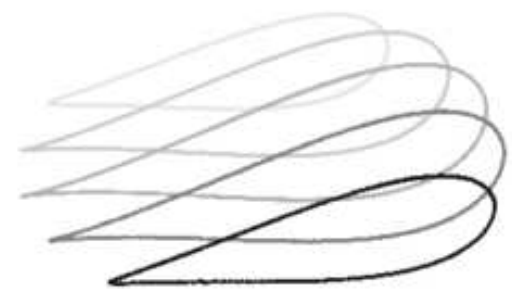

Figure 13: Cuts parallel to the hyperplane $w_{1}=w_{2}$

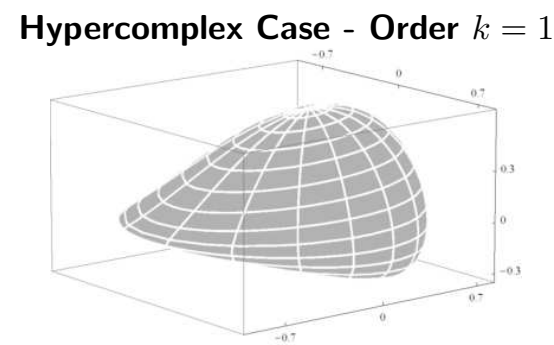

Figure 14: The image of a sphere of radius $\rho=1+|d|$ and center $d=0.15 e_{0}+0.1 e_{1}+0.2 e_{2}$

\section{Complex Case - Order $k=2$}

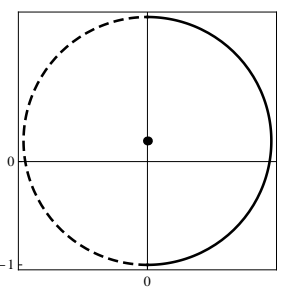

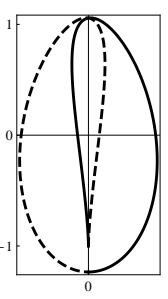

Figure 15: The image of a disk with radius $\rho=1.2$ and center $d=(\rho-1) i$

Hypercomplex Case - Order $k=2$

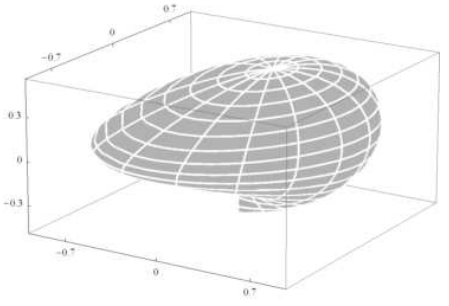

Figure 16: The image of a sphere of radius $\rho=1+|d|$ and center $d=0.1 e_{1}+0.1 e_{2}$

this final remark we leave it as a funny exercise of imagination to the reader to speculate about a possible application of generalized hypercomplex Joukowski transformations in practical circumstances.

Acknowledgments Financial support from "Center for Research and Development in Mathematics and Applications" of the University of Aveiro, through the Portuguese Foundation for Science and Technology (FCT), is gratefully acknowledged. The research of the first author was also supported by the FCT under the fellowship SFRH/BD/44999/2008. Moreover, the authors would like to thank the anonymous referees for their helpful comments and suggestions which improved greatly the final manuscript. 


\section{References}

[1] Barran, M., Hakuri, H.: On two new functional equations for generalized Joukowski transformations. Annales Polon. Math. 56, 79-85 (1991)

[2] Bock, S., Falcão, M. I., Gürlebeck K., Malonek, H.: A 3-Dimensional Bergman Kernel Method with Applications to Rectangular Domains. Journal of Computational and Applied Mathematics, Vol. 189, 67-79 (2006)

[3] Brackx, F., Delanghe, R., Sommen, F.: Clifford Analysis. Pitman 76, London, (1982)

[4] Cruz, J., Falcão, M. I., Malonek H.: 3D-mappings and their approximation by series of powers of a small parameter. In: Gürlebeck, K., Könke, C. (eds.) Proc. 17th Int. Conf. on Appl. of Comp. Science and Math. in Architecture and Civil Engineering, Weimar 10pp.(2006)

[5] De Almeida, R., Malonek, H.R.: On a Higher Dimensional Analogue of the Joukowski Transformation. In: 6th International Conference on Numerical Analysis and Applied Mathematics, AIP Conf. Proc. Vol. 1048, 630-633. Melville, NY, (2008)

[6] De Almeida, R., Malonek, H.R.: A note on a generalized Joukowski transformation. Applied Mathematics Letters. 23, 1174-1178 (2010)

[7] Falcão, M.I, Malonek, H. R.: Generalized exponentials through Appell sets in $\mathbb{R}^{n+1}$ and Bessel functions, In: 5th International Conference on Numerical Analysis and Applied Mathematics, AIP Conf. Proc. Vol. 936, 738-741. Melville, NY, (2007)

[8] Gürlebeck, K., Habetha, K., Spröessig, W.: Holomorphic Functions in the plane and n-dimensional space. Birkäuser, Boston (2008)

[9] Gürlebeck, K., Malonek, H.: A hypercomplex derivative of monogenic functions in $\mathbb{R}^{n+1}$ and its applications. Complex Variables, Theory Appl. 39, 199-228 (1999)

[10] Gürlebeck K., Morais, J.: Geometric characterization of M-conformal mappings, Proc. 3rd Intern. Conf. on Appl. of Geometric Algebras in Computer Science and Engineering AGACSE 17 pp. (2008)

[11] Gürlebeck, K., Morais, J.: On mapping properties of monogenic functions, CUBO A Mathematical Journal, Vol. 11, No. 1, 73-100 (2009)

[12] Gürlebeck, K., Morais, J.: Local properties of monogenic mappings, AIP Conference Proceedings, "Numerical analysis and applied mathematics", In: 7th International Conference on Numerical Analysis and Applied Mathematics, AIP Conf. Proc. Vol. 1168, 797-800. Melville, NY, (2009)

[13] Haruki, H.: A new functional equation characterizing generalized Joukowski transformations. Aequationes Math. 32, no. 2-3, 327-335 (1987)

[14] Kythe, P. K.: Computational Conformal Mapping. Birkhäuser, Boston, (1998)

[15] Kober, H.: Dictionary of Conformal Representations. Dover Publications, New York (1957)

[16] Malonek, H.: A new hypercomplex structure of the euclidean space $\mathbb{R}^{m+1}$ and the concept of hypercomplex differentiability. Complex Variables, Theory Appl. 14, 25-33. (1990)

[17] Malonek, H.: Contributions to a geometric function theory in higher dimensions by Clifford analysis methods: monogenic functions and M-conformal mappings. In: Brackx F., Chisholm, J. S. R., Souček V. (eds.) Clifford Analysis and Its Applications, 213-222. Kluwer, Dordrecht (2001)

[18] Malonek, H.: Selected topics in hypercomplex function theory. In: Eriksson, S.-L. (ed.) Clifford algebras and potential theory, Report Series 7, 111-150. University of Joensuu (2004)

[19] Malonek, H., Falcão, M.I.: 3D-mappings by means of monogenic functions and their approximation. Math. Methods Appl. Sci. 33, 423-430 (2010) 
[20] Väisälä, J.: Lectures on n-dimensional quasiconformal mappings, Lecture Notes in Mathematics, 229, Springer, Berlin (1971)

[21] Aircraft configurations and Wing design, http://en.wikipedia.org/wiki/Blended_wing_body 